\title{
Pairing dynamics in strongly correlated superconductivity
}

\author{
B. Kyung, D. Sénéchal, and A.-M.S. Tremblay \\ Département de physique and Regroupement québécois sur les matériaux de pointe, \\ Université de Sherbrooke, Sherbrooke, Québec J1K 2R1, Canada.
}

(Dated: October 26, 2018)

\begin{abstract}
Confirmation of the phononic origin of Cooper pair formation in superconductors came with the demonstration that the interaction was retarded and that the corresponding energy scales were associated with phonons. Using cellular dynamical mean-field theory for the two-dimensional Hubbard model, we identify such retardation effects in d-wave pairing and associate the corresponding energy scales with short-range spin fluctuations. We find which frequencies are relevant for pairing as a function of interaction strength and doping and show that the disappearance of superconductivity on the overdoped side coincides with the disappearance of the low energy feature in the antiferromagnetic fluctuations, as observed in neutron scattering experiments.
\end{abstract}

In ordinary superconductors, the origin of attraction between electrons, the "pairing glue", manifests itself in observable quantities. Indeed, the characteristic frequencies of phonons appear directly in the frequency dependence of the gap function, which in turn enters observables such as the single-particle density of states or the infrared conductivity. Migdal-Eliashberg theory [1, 2] has been extremely successful to extract from these experiments the spectral function of the phonons that provide the glue.

High-temperature superconductors, heavy-fermion and layered organic superconductors all have phase diagrams where non s-wave superconducting order parameters lie in close proximity to antiferromagnetic phases. In the case of high-temperature superconductors, much effort has been devoted to find out whether antiferromagnetic fluctuations could be the pairing glue $3,3,4,5,6,6]$. Even though its assumptions are not generally valid in that case, Eliashberg theory has been used to extract the amplitude and frequency dependence of a spectral function that is found to be similar to that for antiferromagnetic fluctuations directly measured by neutron scattering $[8,9,10,11]$.

But understanding the origin of pairing in hightemperature superconductors requires an approach that does not rely on the assumptions entering Eliashberg theory and that takes into account Mott insulating behavior. This seems to rule out theories that are based purely on early weak-coupling ideas of boson exchange 12, 13, 14]. In fact, Anderson [15] has argued that the appropriate starting point consistent with Mott physics is the strong-coupling version of the Hubbard model, or the $t-J$ model, and that in this case interactions are instantaneous, as suggested by mean-field factorization [16].

In this paper, we show, for the Hubbard model, that spectral features of the imaginary part of the anomalous (off-diagonal) self-energy do correspond to those of the spectral function for short-range spin fluctuations and that the energy scales relevant for pairing are also those of spin fluctuations.
The Hubbard model Hamiltonian is given by

$$
H=-\sum_{i, j, \sigma} t_{i j} c_{i, \sigma}^{\dagger} c_{j, \sigma}+U \sum_{i} n_{i \uparrow} n_{i \downarrow}
$$

where $t_{i j}$ and $U$ correspond to the hopping matrix and the onsite screened Coulomb repulsion respectively with $c_{i, \sigma}^{(\dagger)}$ the destruction (creation) operators for an electron at site $i$ with spin $\sigma$ and $n_{i \sigma}=c_{i, \sigma}^{\dagger} c_{i, \sigma}$ the number operator. The theoretical method that has been most successful to date to treat the Mott transition starting from the one-band Hubbard model is dynamical mean-field theory (DMFT) 17].

Cluster generalizations of DMFT [18, 19, 20, 21] are necessary to study problems in two dimensions where correlations beyond single site must be taken into account to study, for example, d-wave superconductivity. They lead to phase diagrams that have the same features as those observed experimentally for both electron- and holedoped high-temperature superconductors [22, 23, 24, 25] and for organic conductors. In addition, observable quantities such as the density of states, 24] the ARPES spectrum [23, 24] and the optical conductivity [24] have the experimentally observed behavior. The method that we use, C-DMFT with exact diagonalization at $T=0$, is described in Refs. [19, 23] and in Ref. [26]. We stress that it does not involve any Eliashberg-like approximation.

In C-DMFT, antiferromagnetism and d-wave superconductivity coexist over part of the phase diagram. This is seen in stoichiometric cuprates with intrinsically doped planes [27] and in a few other cases, 28, 29] but does not appear to be a completely generic property of the phase diagram. Our work is thus restricted to showing that spin fluctuations are relevant for high-temperature superconductivity all the way to the overdoped regime, leaving open the possibility that additional types of fluctuations may either contribute to or hinder superconductivity in the underdoped phase.

The correspondence between the imaginary part of the anomalous self-energy, $\Sigma_{a n}^{\prime \prime}$, and the imaginary part of the local spin susceptibility, $\chi^{\prime \prime}$, is seen in Fig. 1. We take band parameters appropriate for $\mathrm{La}_{2-x} \mathrm{Sr}_{x} \mathrm{CuO}_{4}$, 

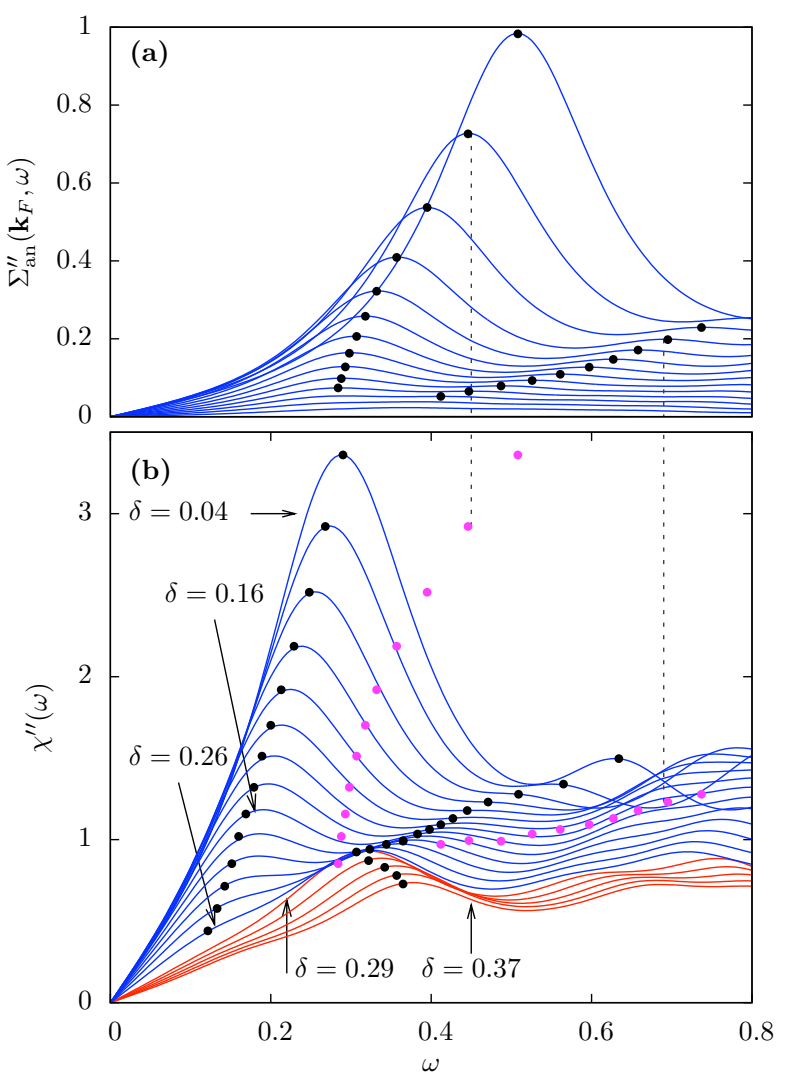

FIG. 1: (Color online) (a) Imaginary part of the anomalous self-energy $\operatorname{Im} \Sigma_{a n} \equiv \Sigma_{a n}^{\prime \prime}$ at the Fermi wave vector nearest to the antinodal point, for various dopings. In (b) Imaginary part of the local spin susceptibility $\operatorname{Im} \chi \equiv \chi^{\prime \prime}$. Black dots in (a) and (b) identify peaks. The position of the peaks of $\Sigma_{a n}^{\prime \prime}$ in (a) are reported as magenta dots in (b) at the same height as the corresponding $\chi^{\prime \prime}$ to illustrate the correspondence between the main peaks of the two functions. The frequency splitting between the peaks decreases with doping, like the single particle gap. The red curves are for the normal state. The lower frequency peak present in the superconducting state disappears and the next peak moves to higher frequency with doping. In all the figures Lorentzian broadening is $0.125 t, U=8 t, t^{\prime}=-0.3 t, t^{\prime \prime}=-0.08 t, t=1, \hbar=1$, for $\mathrm{La}_{2-x} \mathrm{Sr}_{x} \mathrm{CuO}_{4}$.

namely $t^{\prime}=-0.17 t$ for nearest-neighbor and $t^{\prime \prime}=0.08 t$ for next-nearest-neighbor hopping. CDMFT with $U=8 t$ then leads to superconductivity in the doping range observed experimentally [23]. The anomalous self-energy $\Sigma_{a n}^{\prime \prime}$ is defined as minus the off-diagonal part of the inverse Green function in Nambu space. Numerical results are presented in energy units where $t=1$. For all different dopings, the positions of the first two peaks in the spin fluctuations (black dots on middle panel) are just shifted down with respect to the corresponding peaks in $\Sigma_{a n}^{\prime \prime}$ (black dots on top panel).

In Eliashberg theory for the electron-phonon interaction, the first two peaks in the phonon density of states are shifted down with respect to those in $\Sigma_{a n}^{\prime \prime}$ by the
BCS gap [7]. Similarly, the down shift of peaks in $\chi^{\prime \prime}$ seen in Fig. 1b increases as we underdope, like the singleparticle gap. For $U=12 t$ and realistic band structure for $\mathrm{YBa}_{2} \mathrm{Cu}_{3} \mathrm{O}_{7-x}$ the shift is very weakly doping dependent [26].

In Migdal-Eliashberg theory, the real part of the self energy $\Sigma_{a n}^{\prime}$ times the quasiparticle renormalization factor is the gap function. We find that this function, has no static (frequency independent) contribution, 26] contrary to what was found in the $t-J$ model. [7, 24].

To identify the energy scales relevant for the pairs, we introduce the function

$$
I_{G}(\omega) \equiv-\int_{0}^{\omega} \frac{d \omega^{\prime}}{\pi} \operatorname{Im} F_{i j}^{R}\left(\omega^{\prime}\right)
$$

$F^{R}$ is the retarded Gork'ov function defined in imaginary time by $F_{i j} \equiv-\left\langle T c_{i \uparrow}(\tau) c_{j \downarrow}(0)\right\rangle$ with $i$ and $j$ nearestneighbors. The infinite frequency limit of $I_{G}(\omega)$ is equal to $\left\langle c_{i \uparrow} c_{j \downarrow}\right\rangle$ which in turn is proportional to the $T=0$ $\mathrm{d}$-wave order parameter (it changes sign under $\pi / 2$ rotation). It was shown in Ref. [24] that $\left\langle c_{i \uparrow} c_{j \downarrow}\right\rangle$ scales like $T_{c}$. For all these reasons, $I_{G}(\omega)$ is useful to estimate the frequencies relevant for binding. Its meaning is illustrated by the d-wave BCS result in Fig. 2a). The function $I_{G}(\omega)$ rises monotonically until it reaches the sharp BCS cutoff frequency $\omega_{c}$ above which no binding occurs. $I_{G}(\omega)$ extracted from the Eliashberg calculation [30] for lead is also displayed in Fig. 2a). The maximum is reached at a frequency just above the largest phonon frequency.

$I_{G}(\omega)$ is plotted in Fig. 20 for underdoping $\delta=0.4$, optimal doping $\delta=0.16$ and overdoping $\delta=0.26$. The asymptotic large frequency value of $I_{G}(\omega)$ indicated by horizontal lines gives the order parameter that, as a function of doping, has the dome shape dependence [23]. The functions $I_{G}(\omega)$ cross their respective asymptotic values at progressively lower frequencies as doping increases. The spin fluctuations that dominate at the lower frequencies come from wave vectors around $(\pi, \pi)$, as illustrated in Fig. 20 for an underdoped case. The maximum of $I_{G}(\omega)$ is more pronounced in the underdoped regime. The form of $I_{G}(\omega)$ in the overdoped regime is closer to the BCS limit with just a sharp cutoff. Our calculations are less precise at high frequencies, but nevertheless they suggest that, in all cases, $I_{G}(\omega)$ undershoots very slightly its asymptotic value and then recovers at frequencies that are of order $U / 2$ where the upper Hubbard band opens new scattering channels [7]. This has no analog in ordinary superconductors.

In Fig. 3 we focus on the low-frequency behavior. On the top panel, $I_{G}(\omega)$ crosses its asymptotic value for the first time near its maximum. This crossing point shown by vertical lines follows the first peak in the corresponding $\chi^{\prime \prime}$ in the bottom panel. By studying the cases $U=8,12,16$ we have verified that these features scale with $J$. Clearly, if we wished to design an approximate 


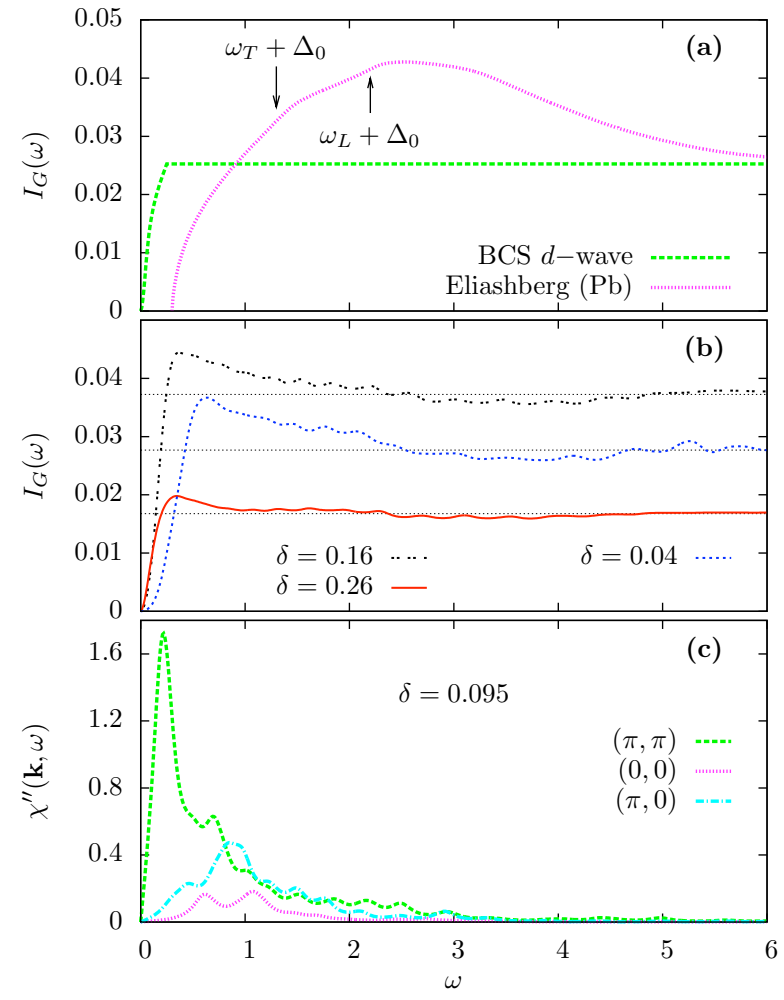

FIG. 2: (Color online) (a) The dashed green line is $I_{G}(\omega)$ for a d-wave BCS superconductor with a cutoff at $\omega_{c}=0.5$. The magenta line is obtained from Eliashberg theory for $\mathrm{Pb}$ in Ref. 30. Frequencies in that case are measured in units of the transverse phonon frequency. The two glitches before the maximum correspond to the transverse and longitudinal peaks in the phonon density of states. The scale of the vertical axis is arbitrary. (b) $I_{G}(\omega)$ calculated for various dopings. The horizontal lines for the asymptotes mark the value of the order parameter. (c) The three independent Fourier components of $\chi^{\prime \prime}$ on a $2 \times 2$ plaquette for an underdoped case. The $(\pi, \pi)$ component dominates at low frequencies.

mean-field theory 31] for this problem that would play a role analogous BSC theory as an approximation of the Migdal-Elisahberg theory, we would use a frequency cutoff of order $J$.

Let us now discuss how the properties of the spin fluctuations $\chi^{\prime \prime}(\omega)$ correlate with those of the d-wave superconducting state for $\mathrm{La}_{2-x} \mathrm{Sr}_{x} \mathrm{CuO}_{4}$. In Fig. 1b, one sees that in the underdoped regime the low frequency peak is the most prominent feature. Optimal doping $(\delta \sim 0.16)$ is reached when the intensity of the low frequency peak becomes comparable to the next one at higher frequency. More importantly one sees that, around doping $\delta=0.26$, superconductivity disappears with the low frequency peak in $\chi^{\prime \prime}(\omega)$ below $\omega$ about $J / 2=0.25 t$. That low frequency peak is the one involved in the dynamics of the pairs as shown by the intersection of the $I_{G}(\omega)$ function with its asymptotic value in Fig. 2 b. The leftover peak in the normal state, indicated in

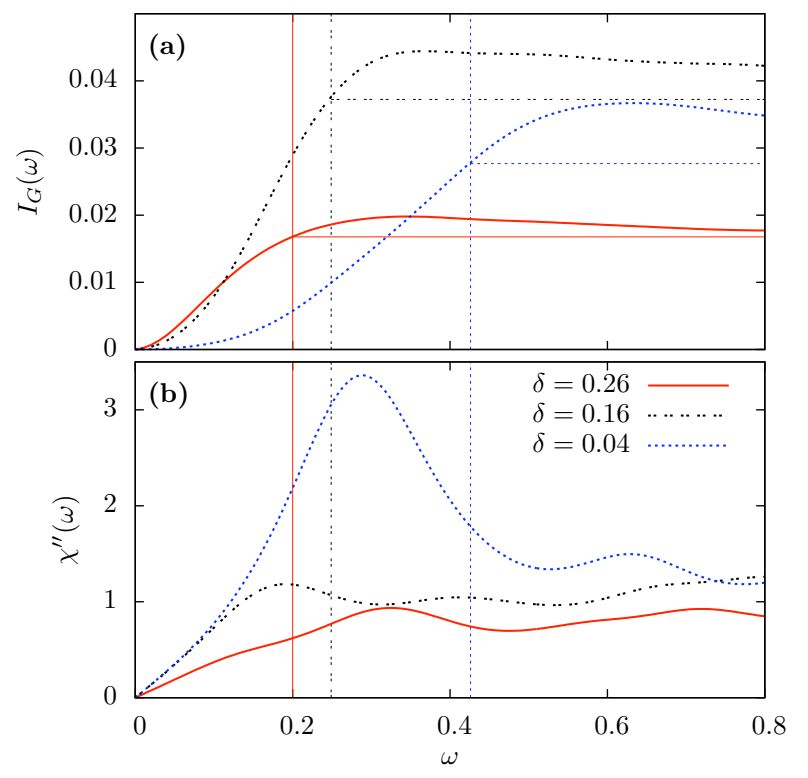

FIG. 3: (Color online) (a) Low frequency part of $I_{G}(\omega)$ for three dopings, underdoped, optimally doped and overdoped. The vertical lines indicate the first intersection with the asymptotic value. The plot of $\chi^{\prime \prime}$ in (b) shows that the first peak occurs in the frequency interval where $I_{G}(\omega)$ reaches its maximum. These are the main frequencies that give rise to pair binding.

red in Fig. 10, moves to higher frequencies as we dope. These features are those found in neutron scattering experiments. 32, 33]

The large tails and monotonic decrease of the weight of the low frequency peak in $\chi^{\prime \prime}$ as we dope are consistent with the "glue function" extracted from recent optical conductivity experiments. [11] The position of the low frequency peak near $0.2 t$ at optimal doping is consistent with the experimental value [1] if we take $t=250 \mathrm{meV}$. One should recall that Fig. 1 $\mathrm{b}$ for the local spin spectral weight $\chi^{\prime \prime}$ gives information integrated in wave vector so the properties of the "neutron resonance" located at $(\pi, \pi)$ have to be found by a different approach. In a recent calculation with a related cluster method, 34] it has been found that the peak located at $(\pi, \pi)$ in the infinite lattice decreases with frequency in the underdoped regime. Given the small weight of this "neutron resonance", this does not contradict the fact that $\chi^{\prime \prime}$, whether local or averaged over one-quarter of the Brillouin zone near $(\pi, \pi)$, has the opposite doping dependence [32, 33, 35, 36, 37] The magnetic resonance itself has small weight [38].

We stress that, despite the similarities, the results obtained in this paper are not identical to those that are obtained in ordinary superconductors. In particular, in the underdoped regime the spin fluctuations are strongly pair-breaking. The pair-breaking effect of the pseudogap can be seen from the fact that in the normal state, the 
pairing susceptibility decreases as one approaches halffilling when vertex corrections are neglected [26]. Following the suggestion of Ref. 39], we also checked whether the pseudogap is pair breaking by computing the ratio $\Sigma_{a n}\left(i \omega_{n}\right) /\left(1-\Sigma_{n}\left(i \omega_{n}\right) / i \omega_{n}\right)$ at the antinodal Fermi surface crossing as a function of Matsubara frequency. The ratio is far from constant, in agreement with the existence of strong pair-breaking effects in the pseudogap. From a diagrammatic point of view, spin fluctuations both scatter electrons (self-energy), decreasing the density of states at the Fermi surface, and provide the glue (vertex). This can lead to the dome shape of the transition temperature as a function of doping [40]. In the underdoped regime, the pair-breaking effect wins over the glue provided by the vertex, whereas in the overdoped regime the vertex dominates.

In conclusion, we have found that the imaginary part of the anomalous self-energy has a structure that is correlated with the spectrum of spin fluctuations. This correlation is similar to the one found with the phonon spectrum in the Migdal-Eliashberg theory of ordinary superconductors. This suggests the importance of spin-one excitations in pair formation. Our approach also allows for mutual feedback between spin fluctuations and pairing [41]. The frequencies most relevant for the pair dynamics scale with the Heisenberg exchange $J$. Superconductivity disappears for sufficient overdoping when the first peak in $\chi^{\prime \prime}$ below frequencies about $J / 2$ becomes negligible. There are, however, major differences with ordinary superconductors. Even though the anomalous self-energy increases as we approach half-filling, the order parameter decreases because of large self-energy effects in the normal part of the propagator. These come from Mott Physics at half-filling. The magnetic fluctuations that we find have the same doping and energy dependence as that found in optical, 9, 10, 11] tunneling [8, 42] and neutron experiments [32, 33, 35, 36, 37]. This work leaves open the possibility that in the underdoped regime there exists other instabilities that compete with antiferromagnetism and d-wave superconductivity.

We are grateful to D.J. Scalapino for insights at the origin of this work, to C. Bourbonnais, J. Carbotte, M. Civelli, T. Maier for discussions, and to M. Greven and G. Kotliar and L. Taillefer for careful reading and comments on the manuscript. This work was supported by NSERC (Canada), CFI (Canada), CIFAR, and the Tier I Canada Research chair Program (A.-M.S.T.). Computations were carried out on clusters of the Réseau québécois de calcul de haute performance (RQCHP) and on the Elix cluster at Université de Sherbrooke. A.-M.S.T. thanks the Aspen Center for Physics.
[1] W. L. McMillan and J. M. Rowell, Phys. Rev. Lett. 14, 108 (1965).

[2] J. P. Carbotte, Rev. Mod. Phys. 62, 1027 (1990).

[3] N. Bickers and D. Scalapino, Phys. Rev. Lett. 62, 961 (1989).

[4] J. Carbotte, E. Schachinger, and D. Basov, Nature 401, 354 (1999), ISSN 0028-0836.

[5] A. V. Chubukov, D. Pines, and J. Schmalian, The Physics of Superconductors (Springer-Verlag, 2003).

[6] P. Monthoux, D. Pines, and G. Lonzarich, Nature 450, 1177 (2007).

[7] T. A. Maier, D. Poilblanc, and D. J. Scalapino, Phys. Rev. Lett. 100, 237001 (2008).

[8] J. F. Zasadzinski, et al., Phys. Rev. Lett. 96, 017004 (2006).

[9] J. Hwang, et al., Phys. Rev. Lett. 100, 137005 (2008).

[10] J. Hwang, et al., Phys. Rev. Lett. 100, 177005 (2008).

[11] E. van Heumen, et al., arXiv:0807.1730v3 (2008).

[12] M. Beal-Monod, et al., Phys. Rev. B 34, 7716 (1986).

[13] D. Scalapino, et al., Phys. Rev. B 34, 8190 (1986).

[14] K. Miyake, et al., Phys. Rev. B 34, 6554 (1986).

[15] P. W. Anderson, Science 316, 1705 (2007).

[16] G. Kotliar and J. Liu, Phys. Rev. Lett. 61, 1784 (1988).

[17] A. Georges, et al., Rev. Mod. Phys. 68, 13 (1996).

[18] M. H. Hettler, et al., Phys. Rev. B 58, R7475 (1998).

[19] G. Kotliar, et al., Phys. Rev. Lett. 87, 186401 (2001).

[20] M. Potthoff, Eur. Phys. J. B (France) 32, 429 (2003).

[21] T. Maier, et al., Rev. Mod. Phys. 77, 1027 (2005).

[22] D. Sénéchal, et al., Phys. Rev. Lett. 94, 156404 (2005).

[23] S. S. Kancharla, et al., Phys. Rev. B 77, 184516 (2008).

[24] K. Haule and G. Kotliar, Phys. Rev. B 76, 104509 (2007).

[25] A. Macridin, et al., Phys. Rev. B 71, 134527 (2005).

[26] B. Kyung, et al., See EPAPS Document No. for supplementary information on methods and additional results. (2009).

[27] H. Mukuda, et al., arXiv:0810.0880v1 (2008).

[28] P. Dai, et al., Phys. Rev. B 71, 100502(R) (2005).

[29] J. Chang, et al., et al., Phys. Rev. Lett. 98, 077004 (2007).

[30] D. J. Scalapino, et al., Phys. Rev. 148, 263 (1966).

[31] P. W. Anderson, et al., J. Phys: Condensed Matter 16, R755 (2004).

[32] S. Wakimoto, et al., Phys. Rev. Lett. 92, 217004 (2004).

[33] S. Wakimoto, et al., Phys. Rev. Lett. 98, 247003 (2007).

[34] S. Brehm, et al., arXiv:0811.0552 (2008).

[35] H. F. Fong, et al., Phys. Rev. B 61, 14773 (2000).

[36] O. J. Lipscombe, et al., Phys. Rev. Lett. 99, 067002 (2007).

[37] B. Vignolle, et al., Nature Physics 3, 163 (2007).

[38] H.-Y. Kee, et al., Phys. Rev. Lett. 88, 257002 (2002).

[39] M. Schiro, et al., Phys. Rev. B 77, 104522 (2008).

[40] B. Kyung, et al., Phys. Rev. B 68, 174502 (2003).

[41] C. Bourbonnais and A. Sedeki (unpublished).

[42] A. N. Pasupathy, et al., Science 320, 196 (2008).

[43] M. Caffarel and W. Krauth, Phys. Rev. Lett. 72, 1545 (1994).

[44] M. Capone, M. Civelli, S. S. Kancharla, C. Castellani, and G. Kotliar, Phys. Rev. B 69, 195105 (2004).

[45] B. Kyung, G. Kotliar, and A.-M. S. Tremblay, Phys. Rev. B 73, 205106 (2006). 


\section{METHODS, SUPPLEMENT}

In Cellular Dynamical Mean-Field Theory (C-DMFT) a cluster is embedded in a bath of non-interacting electrons that simulates the effect of the rest of the infinite lattice by injecting and removing electrons on the cluster with the appropriate single-particle propagator. The bath is determined self-consistently by requiring that the self-energy of the infinite system and that of the cluster be the same. To break the symmetry, frequency independent source fields are allowed on bath sites only. More details can be found in 23]. All the calculations are done with exact diagonalization at zero temperature [43].

For the case of a $2 \times 2$ plaquette, which we shall consider throughout this work, the Nambu spinor is defined by $\Psi_{d}^{\dagger} \equiv\left(c_{1 \uparrow}^{\dagger}, \ldots, c_{4 \uparrow}^{\dagger}, c_{1 \downarrow}, \ldots, c_{4 \downarrow}\right)$, and the greek letters $\mu, \nu=1, \ldots, N_{c}$ label the degrees of freedom within the cluster. We compute the cluster propagator $\widehat{G}_{c}$ by solving the cluster impurity Hamiltonian that will be described shorty. Given the $\hat{\mathcal{G}}_{0}$ on the cluster that results from the presence of the bath, we extract the cluster self energy from $\hat{\Sigma}_{c}=\hat{\mathcal{G}}_{0}^{-1}-\widehat{G}_{c}^{-1}$. Here,

$$
\widehat{G}_{c}\left(\tau, \tau^{\prime}\right)=\left(\begin{array}{cc}
\hat{G}_{\uparrow}\left(\tau, \tau^{\prime}\right) & \hat{F}\left(\tau, \tau^{\prime}\right) \\
\hat{F}^{\dagger}\left(\tau, \tau^{\prime}\right) & -\hat{G}_{\downarrow}\left(\tau^{\prime}, \tau\right)
\end{array}\right)
$$

is an $8 \times 8$ matrix, $G_{\mu \nu, \sigma} \equiv-\left\langle T c_{\mu \sigma}(\tau) c_{\nu \sigma}^{\dagger}(0)\right\rangle$ and $F_{\mu \nu} \equiv$ $-\left\langle T c_{\mu \uparrow}(\tau) c_{\nu \downarrow}(0)\right\rangle$ are the imaginary-time ordered normal and anomalous Green functions respectively.

Using the self-consistency condition,

$$
\hat{\mathcal{G}}_{0}^{-1}\left(i \omega_{n}\right)=\left[\frac{N_{c}}{(2 \pi)^{2}} \int d \tilde{\mathbf{k}} \widehat{G}\left(\widetilde{\mathbf{k}}, i \omega_{n}\right)\right]^{-1}+\hat{\Sigma}_{c}\left(i \omega_{n}\right)
$$

with

$$
\widehat{G}\left(\widetilde{\mathbf{k}}, i \omega_{n}\right)=\left[i \omega_{n}+\mu-\hat{t}(\widetilde{\mathbf{k}})-\hat{\Sigma}_{c}\left(i \omega_{n}\right)\right]^{-1},
$$

we recompute the Weiss field $\hat{\mathcal{G}}_{0}^{-1}$, obtain the corresponding bath parameters by minimizing a distance function described below, and iterate till convergence. Here $\hat{t}(\widetilde{\mathbf{k}})$ is the Fourier transform of the superlattice hopping matrix with appropriate sign flip between propagators for up and down spin and the integral over $\widetilde{\mathbf{k}}$ is performed over the reduced Brillouin zone of the superlattice.

A $2 \times 2$ plaquette is embedded in a bath of noninteraction electrons. To solve the cluster impurity problem, we express it in the form of a Hamiltonian $H_{\mathrm{imp}}$ with a discrete number of bath orbitals coupled to the cluster and use the exact diagonalization technique (Lanczos method) [43] :

$$
\begin{aligned}
H_{\mathrm{imp}} & \equiv \sum_{\mu \nu \sigma} E_{\mu \nu \sigma} c_{\mu \sigma}^{\dagger} c_{\nu \sigma}+\sum_{m \sigma} \epsilon_{m \sigma}^{\alpha} a_{m \sigma}^{\dagger \alpha} a_{m \sigma}^{\alpha} \\
& +\sum_{m \mu \sigma} V_{m \mu \sigma}^{\alpha} a_{m \sigma}^{\dagger \alpha}\left(c_{\mu \sigma}+\text { h.c. }\right)+U \sum_{\mu} n_{\mu \uparrow} n_{\mu \downarrow} \\
& +\sum_{\alpha} \Delta^{\alpha}\left(a_{1 \uparrow}^{\alpha} a_{2 \downarrow}^{\alpha}-a_{2 \uparrow}^{\alpha} a_{3 \downarrow}^{\alpha}+a_{3 \uparrow}^{\alpha} a_{4 \downarrow}^{\alpha}-a_{4 \uparrow}^{\alpha} a_{1 \downarrow}^{\alpha}\right. \\
& \left.+a_{2 \uparrow}^{\alpha} a_{1 \downarrow}^{\alpha}-a_{3 \uparrow}^{\alpha} a_{2 \downarrow}^{\alpha}+a_{4 \uparrow}^{\alpha} a_{3 \downarrow}^{\alpha}-a_{1 \uparrow}^{\alpha} a_{4 \downarrow}^{\alpha}+\text { h.c. }\right) .
\end{aligned}
$$

Here $\mu, \nu=1, \ldots, N_{c}$ label the sites in the cluster and $E_{\mu \nu \sigma}$ represents the hopping and the chemical potential within the cluster. The energy levels in the bath are grouped into replicas of the cluster $\left(N_{c}=4\right)$ (two replicas in the present case) with the labels $m=1, \cdots, N_{c}$ and $\alpha=1,2$ such that we have 16 bath energy levels $\epsilon_{m \sigma}^{\alpha}$ coupled to the cluster via the bath-cluster hybridization matrix $V_{m \mu \sigma}^{\alpha}$. Using lattice symmetries we take $V_{m \mu \sigma}^{\alpha} \equiv$ $V^{\alpha} \delta_{m \mu}$ and $\epsilon_{m \sigma}^{\alpha} \equiv \epsilon^{\alpha}$. The quantity $\Delta^{\alpha}$ represents the amplitude of superconducting correlations in the bath. No static mean-field order parameter acts directly on the cluster sites.

The parameters $\epsilon^{\alpha}, V^{\alpha}$ and $\Delta^{\alpha}$ are determined by imposing the self-consistency condition in Eq. 4 using a conjugate gradient minimization algorithm with a distance function

$$
d=\sum_{\omega_{n}, \mu, \nu}\left|\left(\hat{\mathcal{G}}_{0}^{\prime-1}\left(i \omega_{n}\right)-\hat{\mathcal{G}}_{0}^{-1}\left(i \omega_{n}\right)\right)_{\mu \nu}\right|^{2}
$$

that emphasizes the lowest frequencies of the Weiss field by imposing a sharp cutoff at $\omega_{c}=1.5$. (Energies are given in units of hopping $t$, and we take $\hbar=1$.) The distance function in Eq.(6) is computed on the imaginary frequency axis (effective inverse temperature, $\beta=50$ ) since the Weiss field $\hat{\mathcal{G}}_{0}\left(i \omega_{n}\right)$ is a smooth function on that axis.

With the bond superconducting order parameter defined as

$$
\psi_{\mu \nu}=\left\langle c_{\mu \uparrow} c_{\nu \downarrow}\right\rangle
$$

we consider $d$-wave singlet pairing $\left(\psi \equiv \psi_{12}=-\psi_{23}=\right.$ $\left.\psi_{34}=-\psi_{41}\right)$. The average is taken in the ground state of the cluster.

All quantities depending on wave vector, including selfenergy, are obtained from the Green function periodization scheme.

The finite size of the bath in the exact-diagonalization technique is an additional approximation to the CDMFT scheme. The accuracy of this approximation can be verified by comparing the CDMFT solution for the oneband Hubbard model with the solution from the Bethe ansatz 44. We have also used this comparison in one dimension as a guideline to fix the choice of parameters in the distance function $\left(\omega_{c}=1.5\right.$ and $\left.\beta=50\right)$. These 
results in one dimension also compare well with those obtained using the Hirsch-Fye Quantum Monte Carlo algorithm as an impurity solver where the bath is not truncated [45]. Further, using finite-size scaling for these low (but finite) temperature calculations [45], it was shown that, at intermediate to strong coupling, a $2 \times 2$ cluster in a bath accounts for more than $95 \%$ of the correlation effect of the infinite size cluster in the single-particle spectrum. Because of the finite size of the bath, one also needs to use a finite linewidht broadening $\eta=0.125$ when plotting the figures.

We can also perform an internal consistency check on the effect of the finite bath on the accuracy of the calculation. With an infinite bath, convergence insures that the density inside the cluster is identical to the density computed from the lattice Green function. In practice, we find that there can be a difference of \pm 0.02 between the density estimated from the lattice and that estimated from the cluster. We display results as a function of cluster density since benchmarks with the one-dimensional Hubbard model show that, with a finite bath and the procedure described above, one can reproduce quite accurately Bethe ansatz results for $n(\mu)$ when the cluster density is used. Nevertheless, we should adopt a conservative attitude and keep in mind the error estimate mentioned above.

\section{SUPPLEMENTARY FIGURES AND APPENDICES}

\section{Technical comments on Fig. 1}

The off-diagonal self-energy $\Sigma_{a n}^{\prime \prime}$ is extracted from the periodized Nambu Green function and plotted with $\eta=0.125$. The maxima of the plot are marked with black dots. The imaginary part of the local spin susceptibility $\chi^{\prime \prime}(\omega)$ is calculated on the cluster and plotted with the same value of $\eta$. The dots indicate the maxima in the limit $\eta=0$. The various densities evaluated on the cluster are plotted for the following values of $(\mu, \delta)$ starting from the normal state : $(0.25,0.37)$, $(0.375,0.35), \quad(0.5,0.33), \quad(0.625,0.31), \quad(0.75,0.29)$, $(0.875,0.26), \quad(1.0,0.24), \quad(1.125,0.22), \quad(1.25,0.20)$, $(1.375,0.18), \quad(1.5,0.16), \quad(1.625,0.14), \quad(1.75,0.13)$, $(1.875,0.11), \quad(2.0,0.10), \quad(2.125,0.08), \quad(2.25,0.07)$, $(2.375,0.05),(2.5,0.04)$.

\section{Relation between shift in peak position and single-particle gap}

Fig. 4illustrates how the single-particle gap in the superconducting state $\Delta_{s c}$ (not necessarily the superconducting gap) and the shift $d$ between the position of the peaks in $\Sigma_{a n}^{\prime \prime}$ and $\chi^{\prime \prime}$ change with doping.
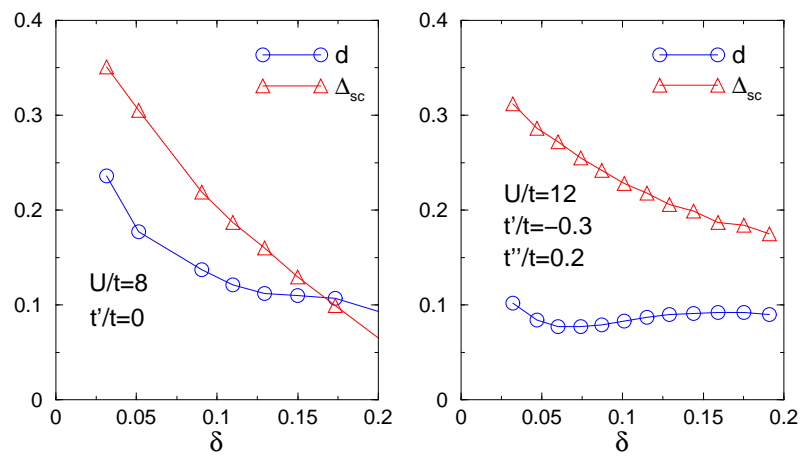

FIG. 4: (Color online) As a function of doping, the shift $d$ (open circles and blue line) between the first peak in the $\chi^{\prime \prime}$ and the first peak in $\Sigma_{a n}^{\prime \prime}$ at the antinodal point. Also shown as a function of doping, the single particle gap $\Delta_{s c}$ (triangles and solid red line) measured from the single-particle density of states. On the left panel, $U=8 t, t^{\prime} / t=0$. On the right panel $U=12 t$ and the band parameters are those appropriate for $\mathrm{YBa}_{2} \mathrm{Cu}_{3} \mathrm{O}_{7-\delta}$.

\section{Real part of the anomalous self-energy}

In conventional Migdal-Eliashberg theory, the real part of the self energy $\Sigma_{a n}^{\prime}$ times the quasiparticle renormalization factor is essentially the gap function. We find that this function, illustrated in Fig 5 , increases as one approaches half-filling, consistent with the increase in the single particle gap found earlier [23] and illustrated in Fig. 4. $\Sigma_{a n}^{\prime}$ has weak frequency dependence near zero frequency only over a range of order $J=4 t^{2} / U$ for $U \gtrsim 8 t$, as can be seen in Fig. 6] If there were a "static" piece to the gap, $\Sigma_{a n}^{\prime}$ would have a frequency independent component at frequencies larger than $J$, at least until frequencies of order $U$. We find that this is not the case. For the $t-J$ model [7, 24] one finds a small instantaneous contribution to $\Sigma_{a n}^{\prime}$, thus making connection with mean-field theories. We show in the main text how mean-field theories can also be seen as approximations to the present approach, even though we do not find an instantaneous contribution to pairing.

$$
I_{G}(\omega)
$$

The Lehman representation for the Nambu Green function allows us to find the following result for the $T=0$ value of $I_{G}(\omega)$

$$
I_{G}(\omega)=\sum_{m}\left\langle 0\left|c_{i \uparrow}\right| m\right\rangle\left\langle m\left|c_{j \downarrow}\right| 0\right\rangle \theta\left(\omega-\left(E_{m}-E_{0}\right)\right)
$$

with $\theta$ the Heaviside step function. Excited states $|m\rangle$ that contribute have an energy less than $\omega$ above the ground state $|0\rangle$.

For BCS s-wave theory, $I_{G}(\omega)$ can be computed ana- 


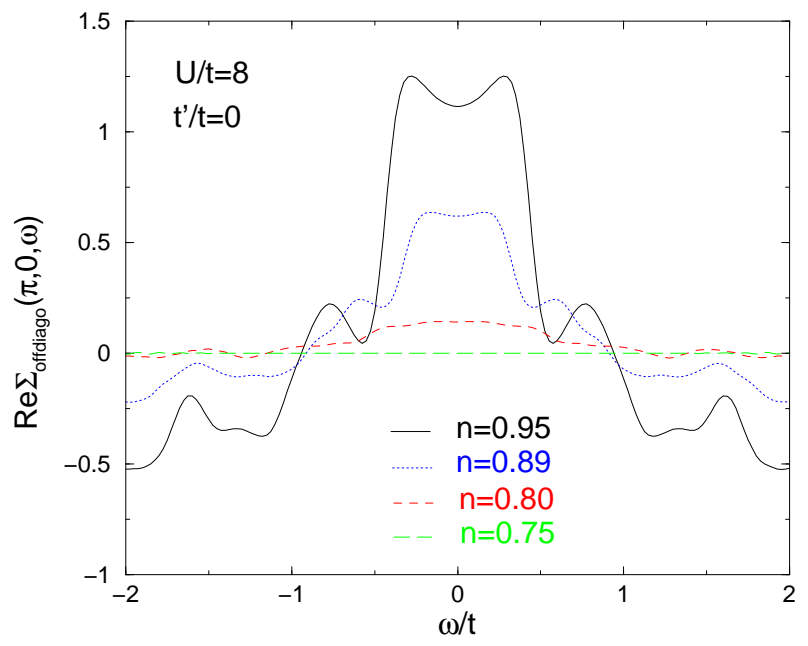

FIG. 5: (Color online) Real part of the anomalous self-energy $\Sigma_{a n}^{\prime}$ for $U=8, t^{\prime}=t^{\prime \prime}=0$ at the antinodal point. Four different dopings are presented. Negative contributions appear at a frequency of order $J$ nearly independent of doping.

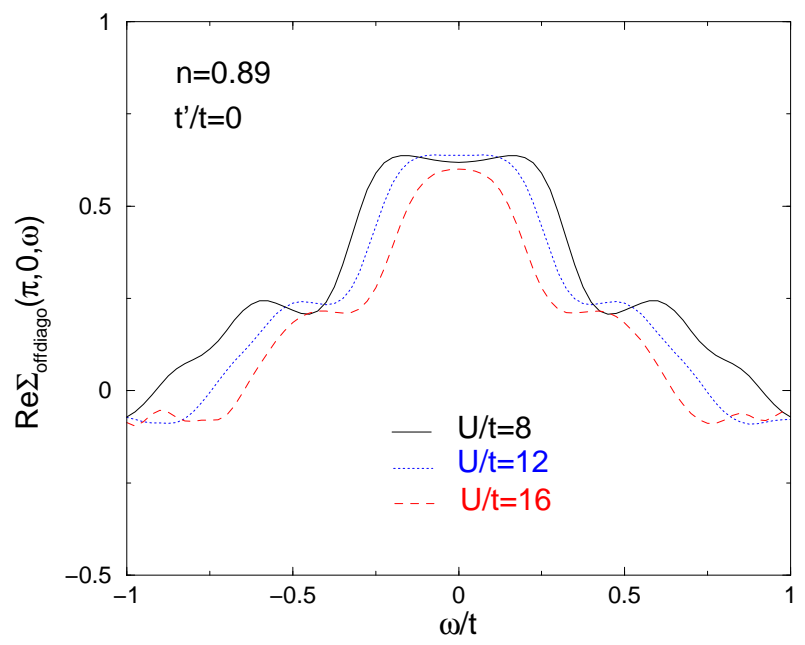

FIG. 6: (Color online) Real part of the anomalous self-energy $\Sigma_{a n}^{\prime}$ as a function of frequency $\omega$ at the antinodal point for fixed doping $\delta=0.11$ and different values of $U=8 t, 12 t$ and $16 t, t^{\prime}=t^{\prime \prime}=0$ represented respectively by solid bleue line, short-dashed red line and long-dashed green line. The nearly flat part near $\omega=0$ decreases with $J$. The range of frequencies where $\Sigma_{a n}^{\prime}$ is positive also decreases as $U$ increases or $J$ decreases.

lytically. One obtains, using $F_{i j}^{R}$ with $i=j$,

$$
\begin{aligned}
I_{G}^{B C S}(\omega) & =\left\langle c_{i \uparrow} c_{i \downarrow}\right\rangle\left[\frac{\sinh ^{-1}(\omega / \Delta)-\sinh ^{-1}(1)}{\sinh ^{-1}\left(\omega_{c} / \Delta\right)-\sinh ^{-1}(1)}\right. \\
& \left.\times \theta(\omega-\Delta) \theta\left(\omega_{c}-\omega\right)+\theta\left(\omega-\omega_{c}\right)\right] .
\end{aligned}
$$

The results for the $d$-wave case in the main text were obtained by numerical integration and a sharp cutoff.

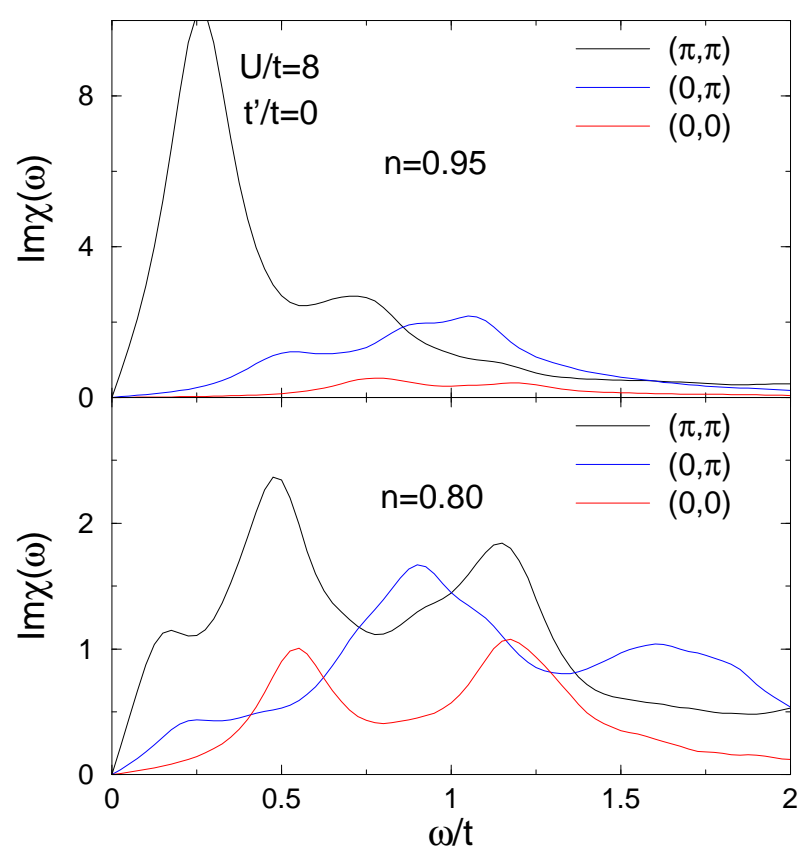

FIG. 7: (Color online) Imaginary part of the spin susceptibility $\chi^{\prime \prime}$ for $U=8 t, t^{\prime}=t^{\prime \prime}=0$ expressed in cluster momenta for underdoping on the top panel $(\delta=0.05)$ and for overdoping on the bottom panel $(\delta=0.2)$. Even in the latter case, a sizable $(\pi, \pi)$ component is left. Recall that the momenta refer to averages over a quarter of the Brillouin zone.

In Eliashbergh theory, the function $I_{G}$ is

$I_{G}(\omega)=N(0) \int_{0}^{\omega} \operatorname{Re}\left[\frac{\Sigma_{a n}\left(\omega^{\prime}\right)}{\sqrt{\left(Z\left(\omega^{\prime}\right) \omega^{\prime}\right)^{2}-\Sigma_{a n}\left(\omega^{\prime}\right)^{2}}}\right] d \omega^{\prime}$

where $N(0)$ is the single-particle density of states at the Fermi level, the square root is in the upper half-plane and

$$
Z(\omega) \equiv 1-\frac{\Sigma_{11}(\omega)+\Sigma_{22}(\omega)}{2 \omega}
$$

with $\Sigma_{i i}(\omega)$ the diagonal components of the self-energy in Nambu space, $\Sigma_{22}(\omega)=-\Sigma_{11}(-\omega)$ and $\Sigma_{a n}(\omega) \equiv$ $\Sigma_{12}(\omega)$. The phase is chosen such that there is no contribution from the second Pauli matrix in Nambu space [30].

The anomalous Green function $F_{i j}^{R}(\omega)$ entering the calculation of $I_{G}(\omega)$ in CDMFT was obtained by Fourier transforming the anomalous lattice Green function calculated with the band Lanczos approach. We used three different values $\eta=0.24,0.18$ and 0.12 for the small imaginary part that must be added to the real frequency to obtain the retarded $F_{i j}^{R}(\omega)$. The final result for $I_{G}(\omega)$ is the extrapolation to $\eta=0$. This is done to smooth the function while preserving as much as possible the asymptotic large frequency value. It differs by only a few per- 


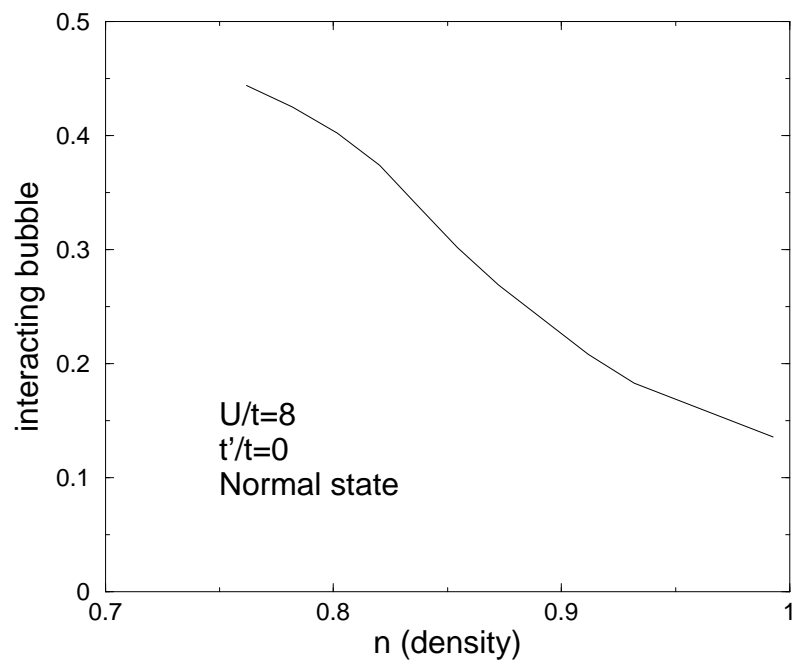

FIG. 8: (Color online) Pairing susceptibility calculated with the dressed bubble only, i.e. without vertex corrections. The decrease near half-filling illustrates the pair-breaking effect of the pseudogap.

cent from the value of the order parameter calculated on the cluster.

\section{Effect of the self-energy in decreasing the pairing tendency as one approaches half-filling}

Fig. 8 shows that in the normal state, the pairing susceptibility calculated without vertex corrections de- creases as one approaches haff-filling. This is an illustration of the detrimental effect of the pseudogap. The self-energy in the dressed Green functions entering the calculation leads to a decrease in the number of states that can pair near the Fermi level.

\section{Attractive Hubbard model}

The cutoff frequency enters very clearly in the integrated off-diagonal spectral weight Eq. (9). Since one expects that the attractive (instead of repulsive) Hubbard model should behave more like the BCS model, we checked that $I_{G}(\omega)$ calculated with C-DMFT for that model does have the structure of the BCS result for $s-$ wave. In other words, it vanishes below the gap, and increases monotonically until a sharp cutoff frequency that depends somewhat on $U$ but is of the order of the bandwidth, as expected from the mean-field solution. There is some structure in the frequency dependence that is probably caused in part by the finiteness of the bath used in the calculation, but does not change the overall trend. 